\title{
Detection of three periodicities in a single oscillating coronal loop
}

\author{
T. Van Doorsselaere ${ }^{1}$, D. C. C. Birtill ${ }^{1}$, and G. R. Evans ${ }^{1}$
}

\begin{abstract}
Centre for Fusion, Space and Astrophysics, Physics Department, University of Warwick, Coventry, CV4 7AL, UK
e-mail: t.van-doorsselaere@warwick.ac.uk
\end{abstract}

Received 23 June 2009 / Accepted 25 October 2009

\section{ABSTRACT}

\begin{abstract}
Context. TRACE $171 \AA ̊$ observations of transverse loop oscillations on May 13, 2001 have been studied by De Moortel \& Brady (2007). They found hints of 3 periodicities present in this event.

Aims. The aim of this article is to improve the analysis, and measure the periodicities more accurately in order to do coronal seismology.

Methods. We create multiple $(x, t)$-slices across the loop, and determine the oscillatory parameters within those slices. We then use statistical methods to reduce the errors on the measured oscillations parameters.

Results. We find two populations with a clearly distinct period. The population at the loop top has a period of $895 \pm 2 \mathrm{~s}$, while the population in the loop leg has a period of $452 \pm 1 \mathrm{~s}$. The fact that the ratio of these two periods is roughly 2 and the geometry of the problem suggests that two harmonics are observed. In the residues, we confirm the presence of a 3rd harmonic with period $309 \pm 15 \mathrm{~s}$ using two separate data analysis techniques. The three periods are then used to do a seismological estimate of the loop expansion and the density scale height.
\end{abstract}

Key words. magnetohydrodynamics (MHD) - Sun: corona - Sun: oscillations

\section{Introduction}

Transverse loop oscillations were first observed by the Transition Region And Coronal Explorer (TRACE) (Aschwanden et al. 1999; Nakariakov et al. 1999). They are manifested as large amplitude oscillations of active region loops with a horizontal polarisation, usually after the occurance of a coronal mass ejection (CME) (Nakariakov et al. 2009). Recently, small amplitude transverse coronal loop oscillations have been observed using the Coronal Multi-channel Polarimeter (CoMP) instrument (Tomczyk et al. 2007), which were interpreted as running kink waves by Van Doorsselaere et al. (2008a). The detection of these small amplitude waves was confirmed with observations using the EUV Imaging Spectrometer (EIS) onboard Hinode (Van Doorsselaere et al. 2008b). With the use of STEREO, the 3D appearance and physics of these waves can now be assessed (Verwichte et al. 2009).

The transverse loop oscillations are perhaps the most studied oscillations in the solar corona. They are the prime target for theoretical (e.g. Ruderman \& Roberts 2002; Andries et al. 2005b; Van Doorsselaere et al. 2008c, 2009) and numerical (e.g. Arregui et al. 2005; Terradas et al. 2006a,b, 2008) modelling efforts and doing coronal seismology (e.g. Nakariakov \& Ofman 2001; Andries et al. 2005a; Arregui et al. 2007). The current observational sample size of such oscillations is very limited, with up to 30 known events (Aschwanden et al. 2002). Because of the high interest, and the limited sample size it is necessary to increase the number of known events, and analyse them with standard techniques.

A few of the events reported in Aschwanden et al. (2002) have been re-analysed with new data analysis techniques: Verwichte et al. (2004); Van Doorsselaere et al. (2007); De Moortel \& Brady (2007). In all of these events, higher harmonics were detected, which were used to determine the vertical structuring in the corona (see Andries et al. 2009, for a review).

In this work, we will re-analyse the loop oscillation studied in De Moortel \& Brady (2007). This event is unique, because the loop oscillation can visually be identified as the 2nd harmonic. This is in contrast with other observational examples, where the fundamental mode dominates the oscillatory signature. The analysis of this unique event could shed a light on the excitation mechanism for these oscillations, which is not well understood.

One popular belief is that these large amplitude kink oscillations are excited by a blast wave emitted by the nearby flare. Such a blast wave has been modelled and proves rather inefficient in exciting the standing oscillation (Terradas et al. 2007). Additionally, this scenario does not explain several key observational features: (1) why is the fundamental mode predominantly excited? (2) What causes the selectivity of the oscillations, so that only a few loops are oscillating? Recently, it has been suggested that vortex shedding caused by an upflow from a CME around the loop could play a role (Nakariakov et al. 2009).

We will analyse the oscillation using the technique employed in Van Doorsselaere et al. (2007). Instead of determining the displacement in a few slices across the loop, we will use all the available information. We will determine the displacements in many slices. This allows to push down the errors on the measurements of the periods, when averaging over the many slits using statistical methods.

Additionally, re-analysing this event allows us to compare the outcomes of different data analysis techniques.

\section{Data analysis}

We study TRACE (Handy et al. 1999) $171 \AA$ data from 02:50 UT until 04:30 UT on the 13th of May 2001. An overview of the 


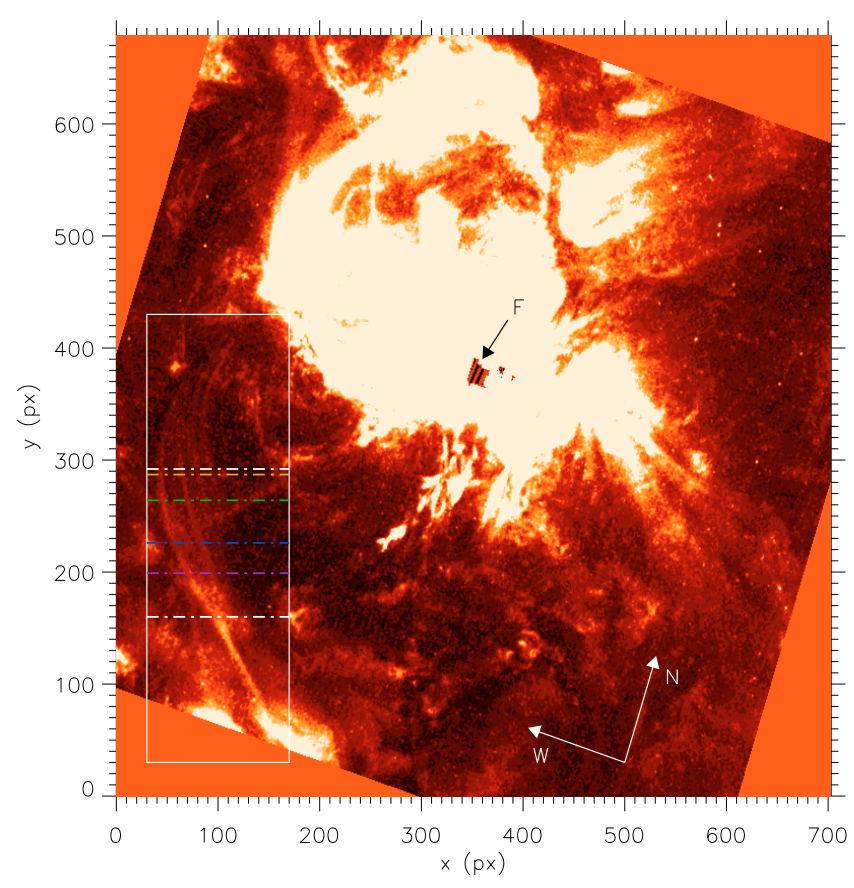

Fig. 1. Overview of the observed loop oscillation. The horizontal and vertical axis are given in pixels (TRACE pixels are .5"). The position of the flare is shown with an "F". The studied loop is enclosed in a box of solid white lines. The extent of the analysed segment of the loop $(160 \mathrm{px} \leq y \leq 292 \mathrm{px})$ is indicated with white dash-dotted lines. Four case studies $y=199,226,264,287 \mathrm{px}$ are marked with purple, blue, green and yellow dash-dotted lines, respectively.

event is given in Fig. 1. In an animation, it can be observed that a flare disrupts the observed active region. The location of the flare is indicated with an "F" in Fig. 1. This flare perturbs the loops in the left hand corner of the image, and oscillations are excited. The loop that will be studied in this article is enclosed in rectangular box of solid white lines. The same loop was also studied by De Moortel \& Brady (2007).

From the previous study and the time series, it can be established that higher harmonics are present in the loop oscillation. The part of the loop below the bottom dashed line in Fig. 1 oscillates in anti-phase with the part above that line. This clearly shows that the loop oscillation is mainly the second harmonic kink mode, as this mode has a velocity node at the loop top.

Other interpretations of the oscillatory phenomenon cannot be excluded. The observed oscillation could be caused by periodic overlapping of optically thin threads. However, such a scenario seems rather unlikely, because the strands would need to be arranged and moving in a specific way (i.e. strands periodically compressing or overlapping) in order to reproduce an apparent period.

The data is pre-processed using trace_prep with the default options. During the observation the field of view is changed a few times. We have removed this jitter by moving the individual frames by the difference in $X$ and $Y$ alignment and a reference point. This is done by looking at the xcen and ycen positions included in the observations header. Only data positions present in all frames are retained for further analysis. We have not corrected the observations for the solar rotation.

Furthermore, the images are rotated over an angle of $18^{\circ}$ in the clockwise direction. This ensures that the line between the loop footpoints is co-aligned with the new $y$-axis. The main aim of this rotation is to have the transverse displacement of the oscillation mainly in the $x$-direction.

To analyse the loop oscillation, the procedure presented in Van Doorsselaere et al. (2007) is followed. The loop oscillation is traced out in multiple $(x, t)$-slices perpendicular to the loop axes and parallel to the transverse motion. This is done by using a semi-automated method for each frame and slice. The user inputs a manual approximation for the position of the loop. This input is taken as the centre of a fitting window in the slice of 20 pixels wide. A Gaussian curve is fitted to the intensity profile across the loop in the slit. The centroid of the Gaussian is used as the position of the loop. This is repeated for many slices. The extent of the loop covered with slices is indicated with horizontal, white, dash-dotted lines in Fig. 1.

For each $(x, t)$-slice, the tracing of the loop results in the transverse position of the loop as a function of time $\left(x_{n}, t_{n}\right)$. Four examples of such a traced out loop are shown in Fig. 2, at the positions indicated with coloured dash-dotted lines in Fig. 1.

To find the oscillation parameters, a Monte-Carlo simulation is performed. Gaussian noise with a standard deviation of $1 \mathrm{px}$ is added to the measured data points. After that, the noisy signal is fitted with a damped sine wave:

$x_{n}=A \sin \left(\frac{2 \pi t_{n}}{P}+\phi\right) \exp \left(-\frac{t_{n}}{\tau}\right)+B+C t_{n}$,

where $A$ is the amplitude of the wave, $P$ the period, $\phi$ the phase, $\tau$ the damping time, $B$ the average $x$-position, and $C$ the trend of the loop motion.

To fit the data, we use the fitting routine mpfit (Markwardt 2009), which uses a Levenberg-Marquardt least-squares fit. It is well known that this fitting routine is very sensitive to the initial estimates. For the period we use $500 \mathrm{~s}$ as initial value, and restrict it between $0 \mathrm{~s}$ and $2000 \mathrm{~s}$. When the fitting results in a boundary value, we reject the fit.

This fitting procedure is performed 1000 times for different noise. For each fitting procedure, a different value of the fitting parameters is obtained, which will result in an ensemble of 1000 values for e.g. the period. The mean of this ensemble is then taken as the best fitting period of the data, and the standard deviation is taken as the error in the period. This gives us a confident estimate of the oscillation parameters, and their respective errors.

The fitted curves are displayed in Fig. 3, for the four cases indicated with dash-dotted lines in Fig. 1 and shown in Fig. 2. It is clear that the fitting method returns satisfactory fits. The only exception is the bad fit for $y=287 \mathrm{px}$ (bottom right panel in Fig. 3), for which the fitting function (Eq. (1)) does not fit the data for any choice of the parameters.

\section{Results}

The results of the curve fitting to the data are presented in Fig. 4, where we plot the relevant fitting parameters and their errors as a function of the slit number $y$.

First we concentrate on the period (shown in the top right panel of Fig. 4). It is clearly visible that two values for the period are obtained. In the region $170 \mathrm{px} \leq y \leq 190 \mathrm{px}$, a period of the order of $900 \mathrm{~s}$ is measured. This region corresponds to the apparent loop top. In the rest of the loop, a different period is measured: approximately $500 \mathrm{~s}$. We have coloured the oscillation parameter measurements according to value of the period; high period measurements are shown with a red $*$, and the lower period measurements are shown with a green $\times$. 

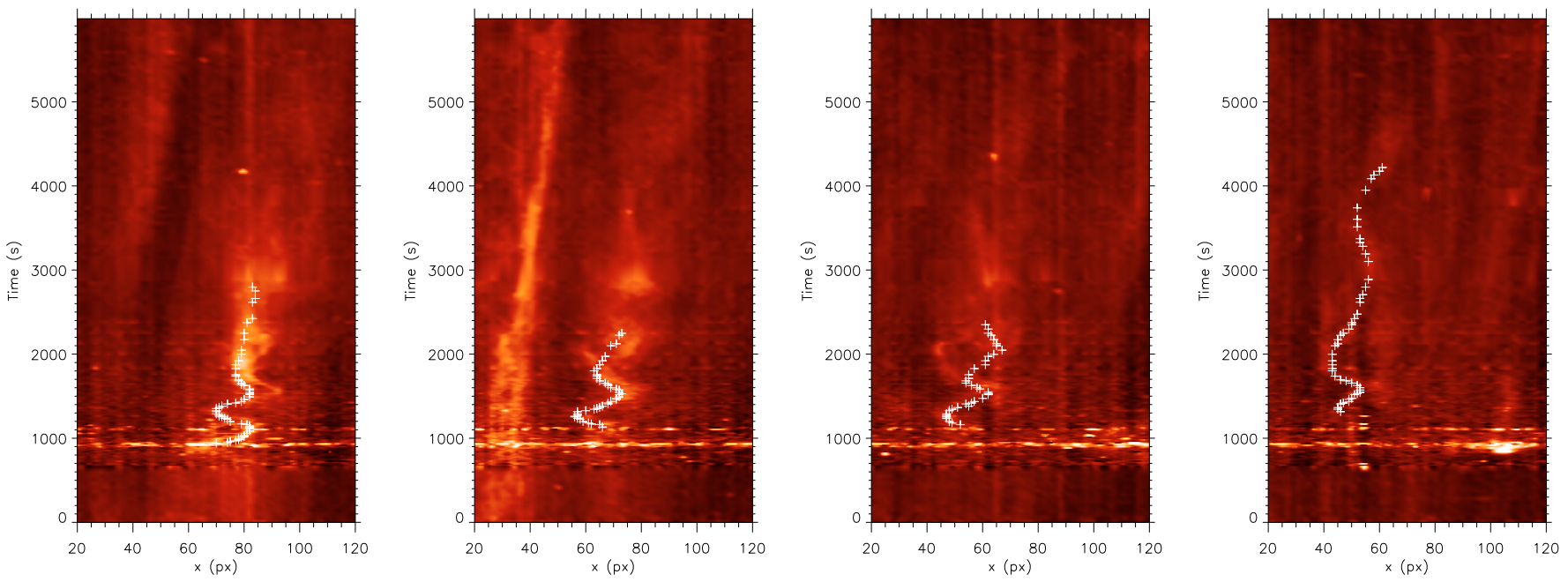

Fig. 2. $(x, t)$-slices at 4 positions in the loop: $y=199$ px (purple dash-dotted line in Fig. 1), $y=226$ px (blue dash-dotted line in Fig. 1), $y=264$ px (green dash-dotted line in Fig. 1), $y=287$ px (yellow dash-dotted line in Fig. 1). The collected points $\left(x_{n}, t_{n}\right)$ are overplotted with white crosses. The vertical axis is time (seconds), while the horizontal axis is TRACE pixels.
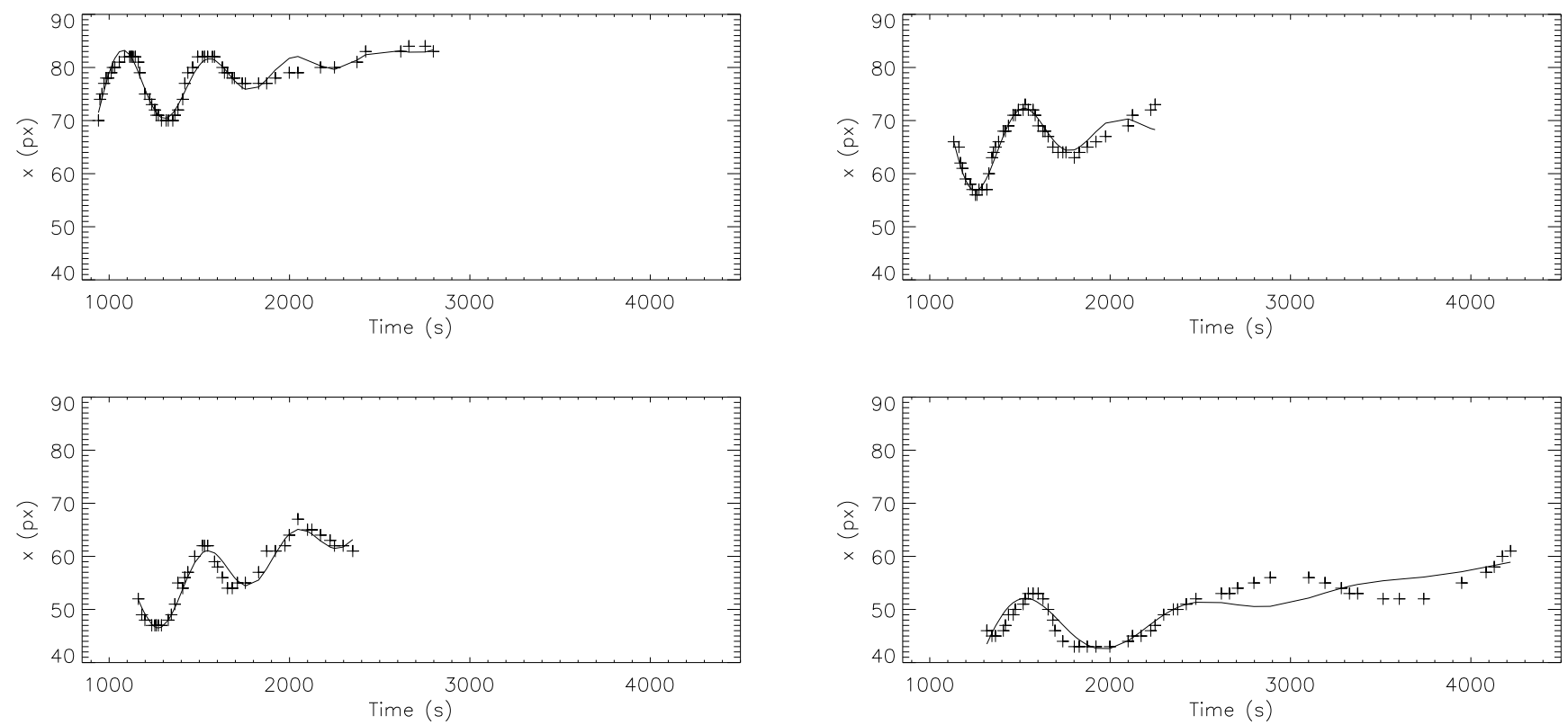

Fig. 3. Fitting curves for the oscillations at the positions shown in Fig. 1 (also shown in Fig. 2). Top left: $y=199$ px (purple dash-dotted line in Fig. 1), top right: $y=226$ px (blue dash-dotted line in Fig. 1), bottom left: $y=264$ px (green dash-dotted line in Fig. 1), bottom right: $y=287$ px (yellow dash-dotted line in Fig. 1). Original data points are shown with a cross (same as in Fig. 2), and the fitted curves are overplotted with a solid line.

The magnitude and location of the two measured periods are indicative of the presence of the fundamental mode and the first overtone. The ratio of the periods is approximately two. The first overtone is observed in the loop legs. The fundamental mode is observed near the loop top, and lower down the loop legs, where it has a higher amplitude than the first overtone. As mentioned before, we use a constant initial value $(500 \mathrm{~s})$ for the period during the fitting. In the loop leg, the fitting routine converges to the first overtone, with a period near the initial value. In the loop top, the fitting routine converges to a much higher period, which means that the lower period is absent near the loop top. The fact that the long period is not detected in the loop leg is caused by the selection of the initial value for the fitting routine. If a higher initial period were chosen, the long period would come out in the loop leg as well.
We use the method mentioned in Van Doorsselaere et al. (2007) to calculate the value of the two periods (their Eqs. (A.1) and (A.2)). For the higher period we obtain $P_{1}=895 \pm 2 \mathrm{~s}$, and for the lower period $P_{2}=452 \pm 1 \mathrm{~s}$. These values of the measured error contain all physical effects. The only effect not included is the shape of the fitting function (Eq. (1)). If a different form would be chosen (e.g. no damping term, time-dependent period), different periods and uncertainties would be obtained.

The ratio of these periods is $P_{1} / P_{2}=1.980 \pm 0.002$. Andries et al. (2005a) showed that the ratio of the periods depends upon the vertical density stratification. The stronger the stratification, the larger the deviation of the ratio from two. On the other hand, the magnetic expansion acts in the opposite direction (Verth \& Erdélyi 2008): the larger the expansion, the higher the period ratio. 

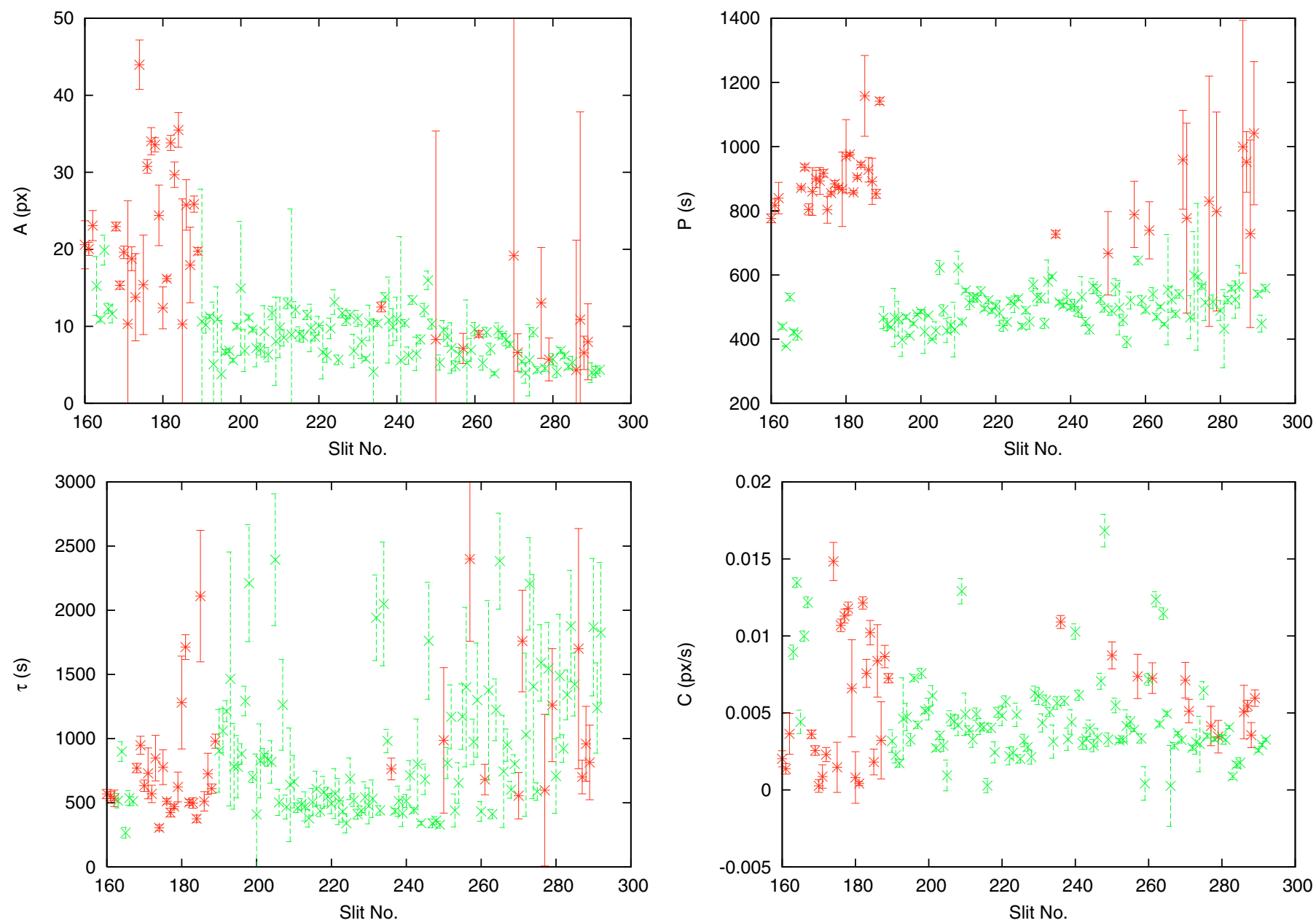

Fig. 4. The results for the fitting parameters in Eq. (1) for each slit (top left: A, top right: $P$, bottom left: $\tau$, bottom right: $C$ ). The results are coloured by their value of the period: red stars have periods over $650 \mathrm{~s}$, while green crosses have a period under $650 \mathrm{~s}$.

The length of this loop has been estimated as $228 \mathrm{Mm}$ by De Moortel \& Brady (2007). This means that the loop extends in the corona up to a height that is comparable to the gravitational density scale height (approximately $50 \mathrm{Mm}$ for the observed wavelength). This tells us that the density variation along the loop can not be neglected. Since the period ratio is almost 2 , however, we can say that the magnetic field expansion will be strong enough to counteract the density stratification. There is no observational evidence of the loop expansion in this data-set.

The damping times are measured to be $\tau_{1}=521 \pm 8 \mathrm{~s}$ and $\tau_{2}=473 \pm 6 \mathrm{~s}$. These damping times are very low compared to the period. This means that the values for the period are mainly determined by the data points at the start of the time series.

From the diagrams with the results for the amplitude (Fig. 4, top left), it can be seen that the red points have a higher amplitude around the loop top (160 px $\leq y \leq 200 \mathrm{px})$, which then drops off further down the loop leg $(y \geq 260 \mathrm{px})$. This amplitude profile is consistent with the fundamental mode. Halfway up the loop leg (200 px $\leq y \leq 260 \mathrm{px}$ ), the second harmonic is discovered, even though it has a lower amplitude than the fundamental mode. The fitting procedure converges to this period, because of the choice of the initial values.

We will now search for periodicities in the residues. The residues are obtained by subtracting the fitted curve (solid line in Fig. 3) from the original data points (crosses in Fig. 3). The same fitting procedure as before is used: Gaussian noise is added
Table 1. The oscillation parameters for the fits to the residue.

\begin{tabular}{cccc}
\hline \hline Slit No. $(\mathrm{px})$ & $A(\mathrm{px})$ & $P(\mathrm{~s})$ & $\tau(\mathrm{s})$ \\
\hline 199 & $0.9 \pm 0.3$ & $288 \pm 24$ & $1300 \pm 700$ \\
226 & $3 . \pm 4$. & $248 \pm 92$ & $375 \pm 1000$ \\
264 & $0.7 \pm 1.6$ & $269 \pm 80$ & $1200 \pm 1000$ \\
287 & $2.7 \pm 0.7$ & $328 \pm 20$ & $600 \pm 600$ \\
\hline
\end{tabular}

to the data, and the fitting function (Eq. (1)) is used. This is repeated 1000 times, to yield average fitting parameters and their standard deviations. The results can be found in Table 1, and the residue together with the fitting function is shown in Fig. 5. The displayed fits seem rather bad, especially in the top and bottom left panels. However, the period which can be visually identified in the data is captured quite well in the first half of the time series. This is particularly the case for the fits in the top left and bottom right panel. In the later half of the observation the fits deteriorate.

Table 1 shows that the fitted periods are all in the same range. If we assume that these periods are a sign of the same oscillation, we can again use the statistical average of the four measurements. We obtain $P_{3}=309 \pm 15 \mathrm{~s}$. As period ratio we obtain $P_{3} / P_{1}=2.90 \pm 0.14$. This period ratio is very close to 3 . This suggests that the observed oscillation in the residue is a third harmonic. 

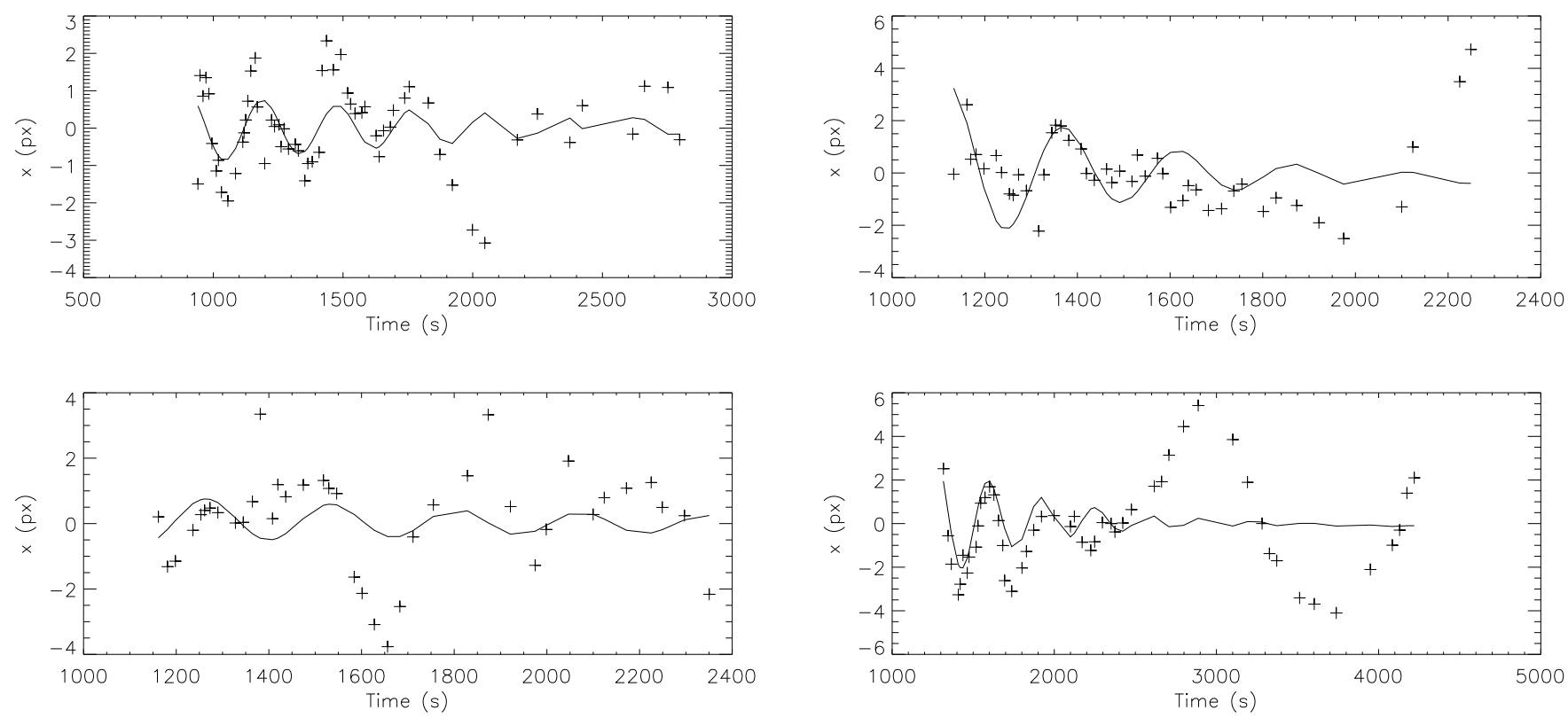

Fig. 5. Fitting curves for the residues at the positions shown in Fig. 1 (also shown in Fig. 2). Top left: $y=199$ px (purple dash-dotted line in Fig. 1), top right: $y=226 \mathrm{px}$ (blue dash-dotted line in Fig. 1), bottom left: $y=264$ px (green dash-dotted line in Fig. 1), bottom right: $y=287 \mathrm{px}$ (yellow dash-dotted line in Fig. 1). The residues are shown with crosses, and the fitted curves are overplotted with a solid line.

It has been suggested (Verth 2007) that using both ratios $P_{2} / P_{1}$ and $P_{3} / P_{1}$ it is possible to determine both the density scale height and the magnetic field variation along the loop. However, quantitative models were not available yet.

Additional indications for the three periods may be found in Scargle periodograms. In the left panel of Fig. 6 we display the Scargle periodogram for the oscillation signal in pixel 199 (purple dash-dotted line in Fig. 1). A broad peak is visible, with pronounced side lobes. To establish that the three spectral peaks are significant, we use a filtration method. We take the highest peak in the left panel, and filter the data for the wave power in that peak (with a spectral window indicated by a dashed line). The filtered signal is then subtracted from the data. The residue is then used to construct the periodogram shown in the middle panel of Fig. 6. Again we use the same filtration method to decide that all three peaks are statistically meaningful.

To construct the periodograms, we interpolated the $\left(x_{n}, t_{n}\right)$ data at pixel 199 to an evenly spaced time sequence. The reason for this is that we use a Fourier transform based frequency filtration method.

From the periodogram, we find periods of $P_{1}^{\prime}=700 \mathrm{~s}$ (range $550 \mathrm{~s}-83 \mathrm{~s}), P_{2}^{\prime}=460 \mathrm{~s}($ range $370 \mathrm{~s}-550 \mathrm{~s})$ and $P_{3}^{\prime}=310 \mathrm{~s}$ (range $290 \mathrm{~s}-340 \mathrm{~s}$ ). These periods have large error bars associated with the width of the spectral peak. Even though the error ranges on these spectral measurements are large, they do not accommodate the values for $P_{1}$ measured with the fitting method. This is not unexpected. The results from the periodograms in fact represent a single measurement in the top right panel of Fig. 4. In that figure, other measurements with large error bars, similar to the ones in the periodogram, are present, which do not necessarily accommodate the eventual ensemble average for the periods $\left(P_{1}, P_{2}\right.$, and $\left.P_{3}\right)$.

The fact that the periods from the fitting are recovered with an entirely unrelated technique (periodograms) is a good indication that they are indeed present in the data. This confirmation is
Table 2. Results for the periods obtained by De Moortel \& Brady (2007).

\begin{tabular}{lccc}
\hline \hline Slit & $P_{i}$ & $P_{i i}$ & $P_{i i i}$ \\
\hline Upper 1 & 1806 & 577 & 301 \\
Upper 2 & 1620 & 606 & 295 \\
Central & 2484 & 672 & 346 \\
Lower & 1038 & 660 & 250 \\
\hline
\end{tabular}

especially needed for the third period, which was detected in the residuals.

\section{Discussion}

\subsection{Comparison with De Moortel \& Brady (2007)}

As discussed before, the studied loop oscillation has been analysed before by De Moortel \& Brady (2007). In their work, De Moortel \& Brady restrict their analysis to 4 slits across the loop. Two above the perceived node in the displacement, one below and one around the node. As in this work, they manually trace out the position of the loop in an $(x, t)$-diagram. After that, they fit the $\left(x_{n}, t_{n}\right)$ points with a fitting function. In contrast to the present work, the fitting function contains 3 harmonics right from the start.

With this method, De Moortel \& Brady find 3 periods in the data, which are given in Table 2 for the different slits they analysed. They interpret the smaller two periods $\left(P_{i i}\right.$ and $\left.P_{i i i}\right)$ as the second harmonic and a higher overtone (possibly the 3rd, 4th or 5th harmonic). We can cross-identify the second harmonic periods, $P_{2}$ in our measurements and $P_{i i}$ in the measurements of De Moortel \& Brady (2007). From such a comparison, it is clear that there is a mismatch in the period of about $30 \%$. The reason for this could be the same as the reason for the discrepancy between the fitting method $P_{2}$ and the periodogram method $P_{2}^{\prime}$ (see previous section), i.e. that the $P_{i i}$-value is only a single measurement of the ensemble used to calculate $P_{2}$. 

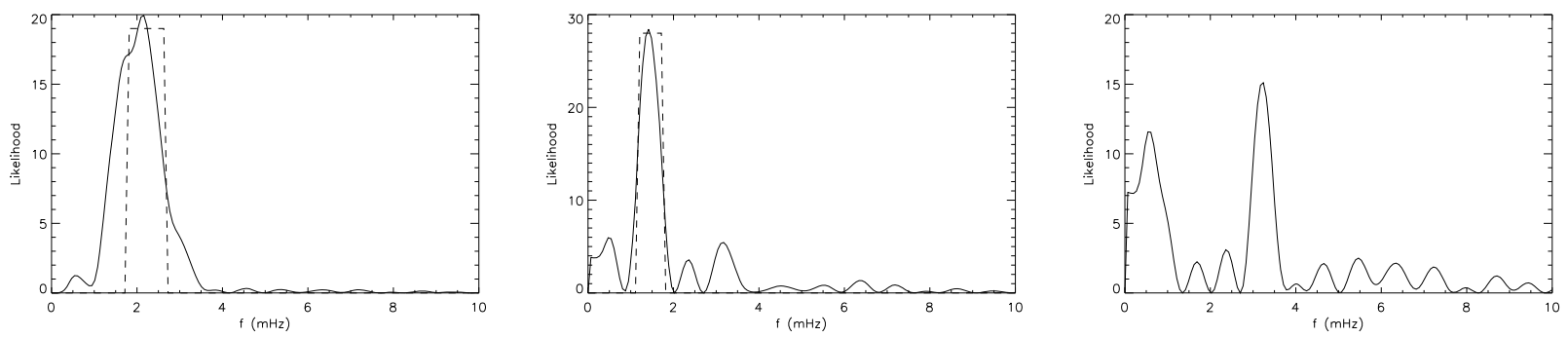

Fig. 6. Periodograms for pixel $y=199 \mathrm{px}$. The left panel shows the periodogram for the original signal. The middle panel shows the periodogram after the filtered signal (with a box indicated by the dashed line, left panel) has been removed. The right panel shows the periodogram after the filtered signal (with a box indicated by the dashed line, middle panel) has been removed.

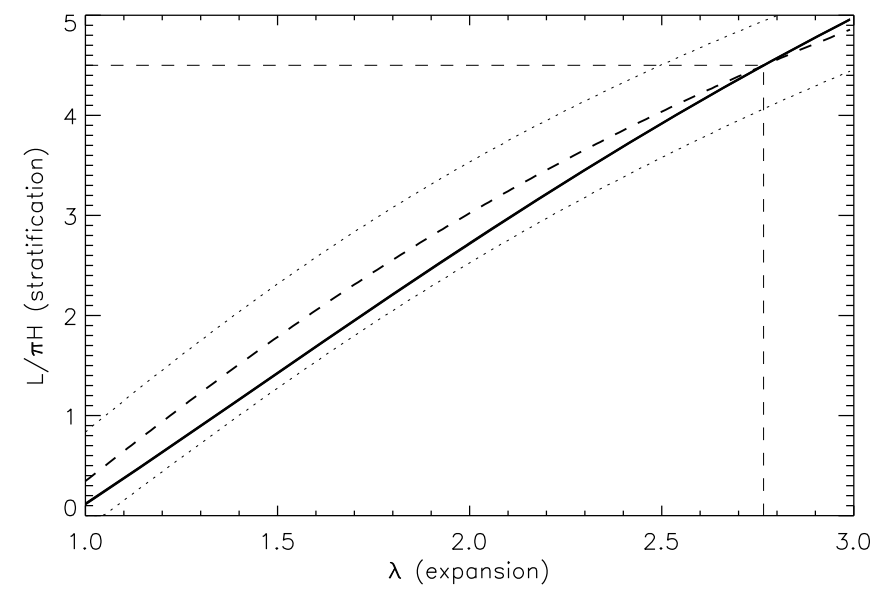

Fig. 7. The thick dashed line and the thick solid line show the solution space for $P_{3} / P_{1}=2.9$ and $P_{2} / P_{1}=1.98$, respectively, in the $(L / \pi H$, $\lambda$ )-plane. The crossing of the two lines is the solution which satisfies the observational constraints, and are indicated with thin dashed lines. The dotted lines show the interval where $P_{3} / P_{1}$ lies between the observational uncertainty estimates. The uncertainty interval for $P_{2} / P_{1}$ is also drawn but is too close to the thick solid line to be visible.

Our measurement of $P_{3}$ agrees very well with the measurement of $P_{i i i}$ by De Moortel \& Brady (2007). Due to the magnitude of the period, and how it compares with the other periods, we have identified this period as the 3rd harmonic.

The value for $P_{1}$ differs more than $50 \%$ with $P_{i}$. The reason for this is unknown. A possible cause is that the period is comparable to the length of the time interval. This means we can only measure one or two periods, and introduces large error bars on this period.

\subsection{Coronal seismology}

As mentioned before, it has been suggested that the detection of three periods may be used to determine the vertical coronal structuring and disentangle the effect of the density stratification and the magnetic field stratification. In this subsection, we will do an attempt to estimate both the loop expansion and the density scale height, using the measured values for $P_{2} / P_{1}$ and $P_{3} / P_{1}$.

As a theoretical model, we use the model by Ruderman et al. (2008), more specifically their equations (67) and (70, corrected for the typo). This model considers a monolithic cylinder as a model for the oscillating loop. However, the observed loop seems to be multi-stranded, with different strands having different phases. Numerically a multi-stranded loop has been considered by Terradas et al. (2008), where they conclude that the loop still shows the same behaviour as a monolithic structure. In the presence of a collection of loops, models were made by Luna et al. (2008); Van Doorsselaere et al. (2008c); Luna et al. (2009) obtaining similar results.

The current model assumes a exponential stratification of the density with a density scale height $H$. Additionally, it assumes the thin tube limit, i.e. that the small radius of the loop is small compared to the loop length. The model allows an expansion of the loop with height, described by the parameter $\lambda=R_{\text {top }} / R_{\text {footpoint }}$ which is assumed to be greater than 1 .

We use the NAG library (d02kef) to numerically solve the Sturm-Liouville problem as described in their paper (Ruderman et al. 2008), for the first three eigenvalues. We repeat this for a parameter range of $1 \leq \lambda \leq 3$ and $0 \leq L / \pi H \leq 5$.

We compute the period ratios over the entire parameter domain, and determine where the numerical period ratios equal the observed period ratios. The result can be found as the thick solid line $\left(P_{2} / P_{1}\right.$ matches $)$ and thick dashed line $\left(P_{3} / P_{1}\right.$ matches $)$ in Fig. 7. The two observational period ratios can only be reproduced (in this model) when the two lines cross, i.e. for $\lambda=2.8$ and $L / \pi H=4.5$ (or $H=16 \mathrm{Mm}$ ). This scale height is extremely small when compared to the commonly believed value of $50 \mathrm{Mm}$ for this temperature range. Moreover, in the observations, no significant expansion of the loop is observed, especially not a factor of (almost) 3 .

When the uncertainty estimates on the period ratios are taken into account, a much better picture is obtained. The region of the parameter space where a period ratio $P_{3} / P_{1}$ is obtained within the observational uncertainty, is outlined with dotted lines. The same is done for $P_{2} / P_{1}$, but the error on that measurement is so small that they visually coincide with the thick solid line. The region where both these uncertainty intervals overlap gives a range of parameters with models that reproduce the observed period ratios. Unfortunately, in this case, the regions overlap everywhere within the dotted lines. This means that the simultaneous measurement of three periods does not help to constrain equilibrium loop parameters in this case, because the uncertainty on the ratio $P_{3} / P_{1}$ is too high.

Note that a crossing for $\lambda \leq 0$ is also possible. We have not studied this, because it is unlikely to have loops that have a smaller radius at the loop top.

We have proven that the measurement of three periods may in theory be used to obtain seismological estimates of the density and magnetic field stratification. From our results, it is clear that the inversion problem is badly conditioned, and the moderate uncertainties on the period ratios may lead to large ranges of possible equilibrium loop parameters.

The fact that the equilibrium parameter solutions can actually be found, shows that the current observation cannot invalidate the theoretical model. 


\section{Conclusions}

We have re-analysed the TRACE observation of coronal loop transverse kink mode oscillations on the 13th of May 2001. Visually, it can be established that the loop is oscillating as the second harmonic kink mode. This event was previously analysed by De Moortel \& Brady (2007). In that work, the authors use a fitting method to find 3 periods, 2 of which they attribute to the second harmonic and a higher harmonic.

We analyse the transverse displacement in many slits across the loop. A Monte-Carlo simulation is used to measure the oscillation parameters of the displacement. For the period, we find two distinct values: a period of $P_{1}=895 \pm 2 \mathrm{~s}$ clustered around the loop top, and a lower period $P_{2}=452 \pm 1 \mathrm{~s}$ in the loop legs. The location of detection of these periods lets us identify them as the fundamental mode and the second harmonic.

After subtraction of the best fit, we find a third period in the residue: $P_{3}=309 \pm 15 \mathrm{~s}$, which we interpret as the 3 rd harmonic. The presence of the third period is confirmed in the Scargle periodogram. This is the first time a 3rd harmonic is detected with confidence.

The values of the periods measured in this work do not agree very well with the results of De Moortel \& Brady (2007), but this may be caused by statistical properties.

We have proven the validity of the proposed method to use the periods of three kink mode harmonics to estimate the density and magnetic field stratification in the corona. We have obtained an expansion factor $\lambda=2.8$ and a density scale height $H=17 \mathrm{Mm}$. These values do not match the observational features of the loop or the commonly believed value for the density scale height. We have shown that the large uncertainties on the period ratios allow for a wide range of equilibrium models, more compatible with the observation.

Acknowledgements. The research leading to these results has received funding from the European Community's Seventh Framework Programme (FP7/20072013) under grant agreement No. 220555.

\section{References}

Andries, J., Arregui, I., \& Goossens, M. 2005a, ApJ, 624, L57

Andries, J., Goossens, M., Hollweg, J. V., Arregui, I., \& Van Doorsselaere, T. 2005b, A\&A, 430, 1109

Andries, J., Van Doorsselaere, T., Roberts, B., et al. 2009, Space Sci. Rev., DOI: $10.1007 / \mathrm{s} 11214-009-9561-2$

Arregui, I., Van Doorsselaere, T., Andries, J., Goossens, M., \& Kimpe, D. 2005, A\&A, 441, 361

Arregui, I., Andries, J., Van Doorsselaere, T., Goossens, M., \& Poedts, S. 2007, A\&A, 463, 333

Aschwanden, M. J., Fletcher, L., Schrijver, C. J., \& Alexander, D. 1999, ApJ, 520,880

Aschwanden, M. J., De Pontieu, B., Schrijver, C. J., \& Title, A. M. 2002, Sol. Phys., 206, 99

De Moortel, I., \& Brady, C. S. 2007, ApJ, 664, 1210

Handy, B. N., Acton, L. W., Kankelborg, C. C., et al. 1999, Sol. Phys., 187, 229

Luna, M., Terradas, J., Oliver, R., \& Ballester, J. L. 2008, ApJ, 676, 717

Luna, M., Terradas, J., Oliver, R., \& Ballester, J. L. 2009, ApJ, 692, 1582

Markwardt, C. B. 2009, in ASP Conf. Ser., ed. D. A. Bohlender, D. Durand, \& P. Dowler, 411,251

Nakariakov, V. M., \& Ofman, L. 2001, A\&A, 372, L53

Nakariakov, V. M., Ofman, L., DeLuca, E. E., Roberts, B., \& Davila, J. M. 1999, Science, 285, 862

Nakariakov, V. M., Aschwanden, M. J., \& Van Doorsselaere, T. 2009, A\&A, 502, 661

Ruderman, M. S., \& Roberts, B. 2002, ApJ, 577, 475

Ruderman, M. S., Verth, G., \& Erdélyi, R. 2008, ApJ, 686, 694

Terradas, J., Oliver, R., \& Ballester, J. L. 2006a, ApJ, 642, 533

Terradas, J., Oliver, R., \& Ballester, J. L. 2006b, ApJ, 650, L91

Terradas, J., Andries, J., \& Goossens, M. 2007, A\&A, 469, 1135

Terradas, J., Arregui, I., Oliver, R., et al. 2008, ApJ, 679, 1611

Tomczyk, S., McIntosh, S. W., Keil, S. L., et al. 2007, Science, 317, 1192

Van Doorsselaere, T., Nakariakov, V. M., \& Verwichte, E. 2007, A\&A, 473, 959

Van Doorsselaere, T., Nakariakov, V. M., \& Verwichte, E. 2008a, ApJ, 676, L73

Van Doorsselaere, T., Nakariakov, V. M., Young, P. R., \& Verwichte, E. 2008b, A\&A, 487, L17

Van Doorsselaere, T., Ruderman, M. S., \& Robertson, D. 2008c, A\&A, 485, 849

Van Doorsselaere, T., Verwichte, E., \& Terradas, J. 2009, Space Sci. Rev., DOI: $10.1007 / \mathrm{s} 11214-009-9530-9$

Verth, G. 2007, Astron. Nachr., 328, 764

Verth, G., \& Erdélyi, R. 2008, A\&A, 486, 1015

Verwichte, E., Aschwanden, M. J., Van Doorsselaere, T., Foullon, C., \& Nakariakov, V. M. 2009, ApJ, 698, 397

Verwichte, E., Nakariakov, V. M., Ofman, L., \& Deluca, E. E. 2004, Sol. Phys., 223,77 